
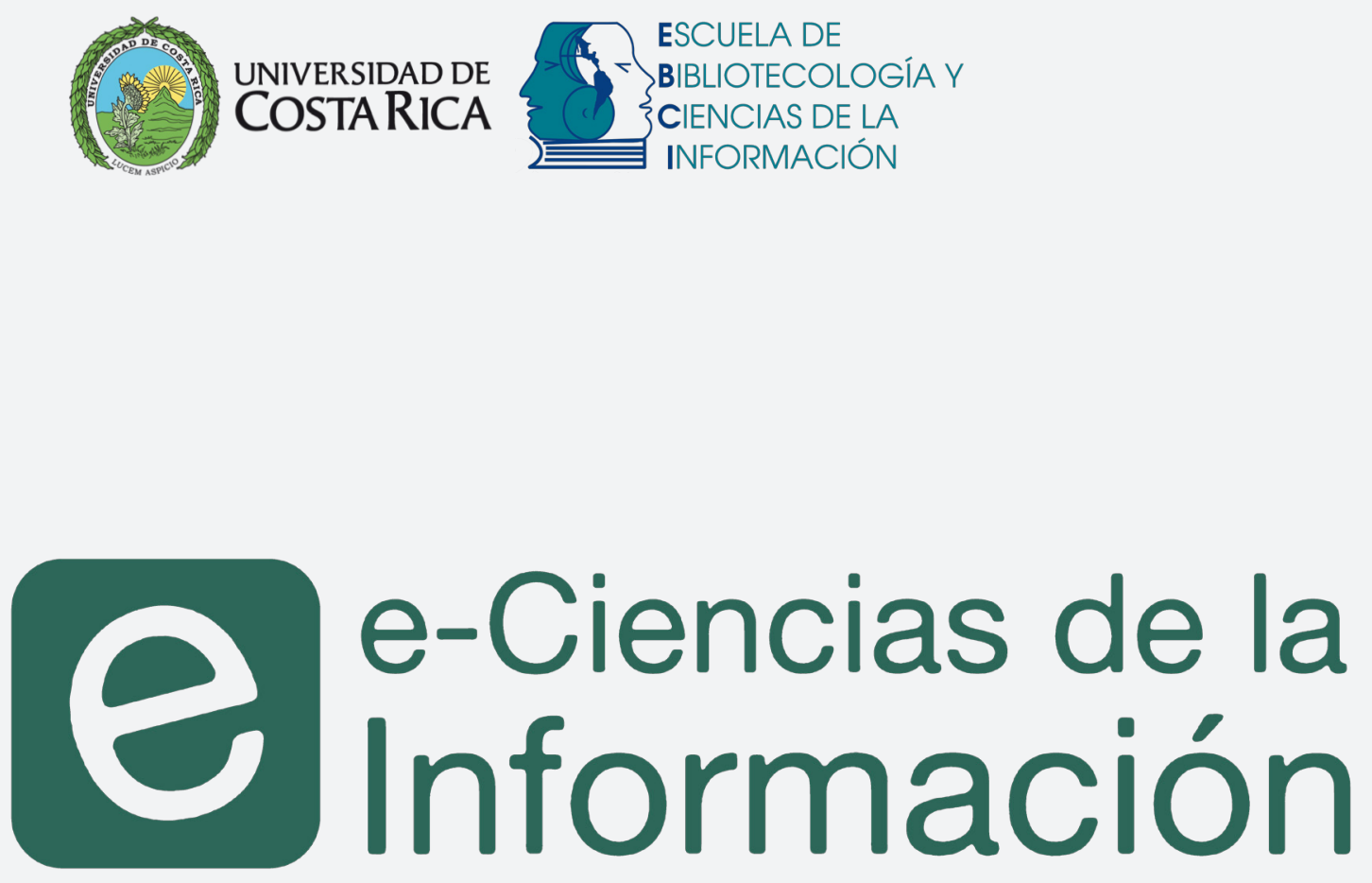

\title{
La Sección de temas locales de la Biblioteca Pública Municipal de Simat de la Valldigna: un testimonio de la historia pasada y presente Lorena Chaves Salgado
}

DOI: https://doi.org/10.15517/eci.v8i1.29926

e-Ciencias de la Información, volumen 8, número 1, Ene-Jun 2017

ISSN: $1649-4142$

\section{다(1)(2)(2)}




\section{La Sección de temas locales de la Biblioteca Pública Municipal de Simat de la Valldigna: un testimonio de la historia pasada y presente} The Local Issues Section of the Municipal Public Library of Simat de la Valldigna: A testimony of past and present history

Lorena Chaves Salgado ${ }^{7}$

\section{RESUMEN}

La biblioteca pública cumple una labor de formación permanente en el campo del saber y la cultura, uno de sus objetivos principales es satisfacer las necesidades de la comunidad en la cual se encuentra inmersa, por medio de la oferta de distintos servicios siendo uno de ellos la sección de temas locales, encargada de brindar a las personas toda aquella información relativa a la historia comunal en sus distintas manifestaciones. Por lo anterior, este trabajo expone la importancia del aporte y la proyección social que presta dicha sección contextualizado su trabajo en la población de Simat de la Valldigna en Valencia, España, se describen, además, por medio a través de ejemplos distintos materiales que conforman este acervo de información. Se concluye poniendo de manifiesto la responsabilidad que tiene la biblioteca pública en lo relativo a mantener viva la historia local de Simat como un aporte a la preservación de la memoria de sus antepasados.

Palabras Clave: biblioteca pública; acción social; comunidades; historia local.

\section{ABSTRACT}

The public library fulfills a permanent formative role in the field of knowledge and culture, one of its main objectives is to meet the needs of the community in which it is immersed. Through the provision of various services, one of them being the local issues section, which is responsible for providing citizens with all the information related to the history of the community in its different manifestations. This paper exposes the importance of the contribution and social projection provided by this section contextualizing its work in the population of Simat de la Valldigna in Valencia, Spain. There are also described, by means of examples, different materials that make up this information reservoir. It concludes by highlighting the responsibility of the public library in keeping alive the local history of Simat as a contribution to the preservation of the memory of their ancestors.

Keywords: public library; social envolment; communities; local history.

1 Universidad de Costa Rica. Escuela de Bibliotecología y Ciencias de la Información. COSTA RICA. https:// orcid.org/0000-0002-3004-1685. lorena.chaves@ucr.ac.cr 


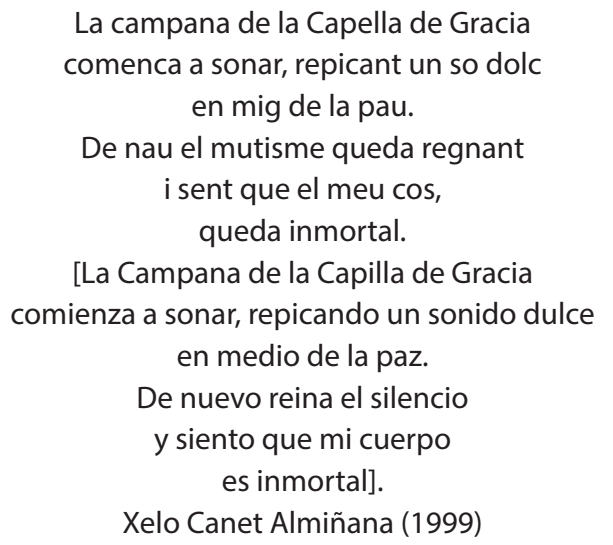

\section{INTRODUCCIÓN}

La Biblioteca pública dinamiza el desarrollo social, respondiendo de forma activa y eficaz a las demandas de la comunidad, colaborando con otras instituciones, grupos de poblaciones especiales y fuerzas vivas de la misma. Debe abrir sus puertas y asumir el papel que le corresponde en el ámbito de la educación, la enseñanza, la formación y la difusión de los recursos culturales del país. Además, dirigir sus esfuerzos hacia el conocimiento y el aprendizaje, para guiar a las personas a tomar, de forma independiente, decisiones que mejoren su calidad de vida a nivel integral.

En ese sentido, entre las diferentes funciones de la Biblioteca pública está contribuir con el desarrollo del patrimonio cultural y artístico de la comunidad, lo que constituye una labor muy importante para conocer y diseminar el conocimiento local, lo anterior se expresa en las directrices IFLA/UNESCO del 2001 por la Federación Internacional de Asociaciones de Bibliotecarios y Biblioteca (por sus siglas en inglés: IFLA) y la Organización de las Naciones Unidas para la Educación, la Ciencia y la Cultura (UNESCO):

La biblioteca pública debe ser una institución fundamental de la comunidad en la que se encuentra en lo que se refiere al acopio, la preservación y la promoción de la cultura local en todas sus modalidades. Puede hacerlo de diferentes maneras, por ejemplo, manteniendo los fondos relativos a la historia del lugar, organizando exposiciones y narraciones orales, editando publicaciones de interés local y creando programas interactivos sobre temas locales. Cuando la tradición oral sea un importante método de comunicación, la biblioteca pública deberá fomentar su continuación y expansión. (2001, p. 14)

En el caso de la comunidad de Simat de la Valldigna en Valencia, España, la Biblioteca pública realiza un importante esfuerzo al resguardar y dar a conocer la variedad de materiales que contiene la colección de temas locales. Ahí es posible encontrar diferentes objetos, desde una trompeta para anunciar actividades de la comunidad hasta una fotografía de finales del siglo XVIII o un pasaje de autobús de los años 50; e. Esta sección, asimismo, es consultada tanto por investigadores que realizan distintos trabajos sobre la historia local de la comunidad, como por políticos cuando hay campañas electorales por su deseo de estar bien documentados sobre hechos históricos. 
También, concurren estudiantes escuela y de colegio para la elaboración de proyectos de investigación, así como universitarios y los ciudadanos de la comunidad que desean informarse sobre distintos temas que les son de interés desde un punto de vista local; en este sentido, la sección local cumple la función de informar y hacer accesible al público una colección de materiales diversos que constituyen una vía de entrada a todo tipo de información y a una diversidad de materiales que, en su conjunto, reconstruyen lo cultural que vienen a aportar conocimiento del pasado de esta localidad.

Por lo anterior, el presente informe técnico expone la importancia del trabajo que realiza la Biblioteca Pública de Simat de la Valldigna con su sección de temas locales, que brinda información en todo lo relativo a costumbres y tradiciones pasadas y también las más cercanas. Conformando, así, un patrimonio de la memoria histórica de la población a la cual representa, revelando un libro abierto con una enorme variedad de temas todos ligados a la vida cotidiana, al pasado vivo de una comunidad que aspira a salvaguardar el testimonio de sus generaciones pasadas.

\section{BREVE RESEÑA HISTÓRICA DE SIMAT DE LA VALLDIGNA}

Esta comarca está rodeada por las montañas de la sierra de Corbera al norte y las del Mondúver al sur, y su población se asienta en un pequeño valle circundado de montañas que queda abierto por el este hacia el mar. Se accede por el litoral desde la población de Tavernes de la Valldigna, o por el interior desde Alzira o desde Xàtiva. La principal actividad económica del municipio es el cultivo de cítricos que cuenta con diferentes almacenes dedicados a la comercialización de la naranja, los cuales dan trabajo a casi toda la población.

La evolución histórica de la población de Simat de la Valldigna está muy ligada a la construcción del Real Monasterio de Santa María de la Valldigna, monumento fundado hacia finales del siglo XIII, que tuvo un importante papel en el desarrollo de la cultura local. Este monasterio fue fundado por la orden monástica francesa, católica reformada del Císter, cuyo origen se remonta al 1098. La Valldigna, antes llamada la Vall d' Alfancech, o Marinyen, fue habitada durante la Alta Edad Media por musulmanes, y conquistada en 1242-43, por el Rey Jaume I. Su nieto, Jaume II de Aragón, en ocasión de una expedición al reino moro de Murcia, al pasar por la Vall de Marinyen, la denominó con el nombre de la Valldigna.

Inicialmente, en la Valldigna convivían cristianos y musulmanes, quienes trabajaban las tierras cedidas en usufructo por el abad del monasterio, aun cuando las condiciones eran más duras para los mudéjares que deseaban continuar con la práctica de su religión, estos se reunían en la mezquita de la Xara para practicar su religión, que también era un lugar de enseñanza, de formalización de contratos y a menudo la sede de justicia de los cadís (legisladores de justicia o jueces musulmanes), la convivencia que mantenían llegó a su fin en el año 1609, con la expulsión de los mudéjares.

La vida en la Valldigna continuó con el desarrollo de la sociedad feudal valenciana, bajo el dominio del Abat del Monasterio hasta finales del siglo XIX. En 1835, con su desamortización, finalizaría el señorío del Abad sobre las tierras de la Valldigna, dando paso a la ruina del monasterio, que pasó a 
manos particulares y permaneció así hasta 1991. Cuando fue adquirido por la Generalitat Valenciana comenzó un proceso de restauración y recuperación de las instalaciones, para dar al monasterio el realce artístico y arquitectónico de esta edificación tan representativa. Lo anterior se evidencia en el artículo 57 del estatuto de Autonomía de la Generalitat Valenciana:

El Real Monasterio de Santa María de la Valldigna es templo espiritual, histórico y cultural del antiguo Reino de Valencia, y es, igualmente, símbolo de la grandeza del Pueblo Valenciano reconocido como Nacionalidad Histórica. La Generalitat recuperará, restaurará y conservará el monasterio, y protegerá su entorno paisajístico. Una Ley de Les Corts determinará el destino y utilización del Real Monasterio de Santa María de la Valldigna como punto de encuentro de todos los valencianos, y como centro de investigación y estudio para recuperar la historia de la Comunitat Valenciana (Ley Orgánica 5/1982, 2016, bloque 87).

Según crónicas de la época, es importante destacar los esfuerzos realizados por impulsar las prácticas culturales de la población, por medio de la creación de la primera biblioteca. En la crónica de 1915 de José Ripoll Mansanet, en 1894 se instala en Simat el primer "Casino Cultural Recreativo", propiedad de Vicente Ripoll Baldés, donde se realizaban bailes y estaba instalada una Biblioteca: "el Casino de la Plaza con su Biblioteca y sus bailes familiares fue el primer paso de cultura popular que dio el pueblo." (Ripoll, 2015, p. 67)

Como referencia a otras prácticas culturales señaladas por el historiador Ripoll Mansanet, cabe mencionar el impulso que se le dio al teatro, de hecho, la primera representación de la que se tiene noticia tuvo lugar en 1886 en el salón parroquial, y fue patrocinada por el entonces cura párroco Don José Serrano; contribuyeron también a impulsar esta práctica: Pepe-Antoni Brines, Leonardo Mansanety el maestro Don Samuel Girau entre otros. En el transcurso del siglo XX se producen, igualmente, algunos acontecimientos destacados que incidieron en el desarrollo social de la población de Simat, como por ejemplo en 1903, cuando se inaugura el primer servicio de electricidad, y en 1912 se instala el primer teléfono interurbano, representando un gran avance, y una comunicación más fluida hacia otras localidades.

En síntesis, Simat es un municipio que cuenta con lugares de mucho interés, rodeado de montañas, bellos parajes, campos de naranjos y una historia íntimamente relacionada con el Monasterio de Santa María de la Valldigna. Sus fiestas patronales en honor a los Santets de la Pedra, a Nuestra Señora de Gracia y al Santísimo Cristo de la Fe conforman, respectivamente, una confluencia de historia y tradición cultural de un pueblo que disfruta conservando la autenticidad de su tierra y de su gente.

\section{CONFORMACIÓN DE LA BIBLIOTECA PÚBLICA DE SIMAT DE LA VALLDIGNA}

En España hubo dos organismos que impulsaron la creación y el ordenamiento de las bibliotecas públicas, el Patronato de Las Misiones Pedagógicas y la Junta de Intercambio y Adquisición de Libros. Mientras la primera efectuaba sus actividades en poblaciones pequeñas, la segunda se ocupaba de municipios más grandes. Las misiones pedagógicas fueron fruto de una serie de iniciativas, una de las más importantes fue el influjo que ejerció la Escuela 
Nueva fundada en 1910, de orientación socialista.

Se destaca el trabajo llevado a cabo por la llamada Institución Libre de Enseñanza, creada en 1876 por Francisco Giner de los Ríos y promovida por una burguesía culta y progresista, que desarrolló un amplio programa de extensión cultural, que contaba con la creación de bibliotecas, fomento a la lectura, organización de conferencias, teatro popular y publicación de boletines, entre otras. Se llevaban a cabo, también, actividades de educación ciudadana para dar a conocer la Constitución, y sesiones de orientación pedagógica para los maestros de escuelas rurales que pretendían mejorar la calidad de la enseñanza.

Inmerso en este contexto, la figura de las bibliotecas vuelve a tomar importancia después de la aprobación del Decreto del 7 de agosto de 1931, que establecía una obligatoriedad para las escuelas primarias de contar con una biblioteca que prestara servicios tanto a los niños como a los adultos, y que debía estar dirigida por un maestro, quien recibía directrices del Consejo Local de Primera Enseñanza. A cada biblioteca que estuviera en una escuela se le daba una caja que contenía una colección de 100 volúmenes, boletas para los préstamos, fichas para realizar las estadísticas, papel para forrar libros y un manual para el cuidado de los mismos; dichas colecciones contenían, entre otros objetos, libros de geografía, de literatura española y universal, manuales agrícolas, biografías, guías de viaje y diccionarios. La colección contenía, de igual manera, cuentos para niños, libros de aventuras y obras de referencia para realizar consultas sobre distintos temas.

Es importante destacar, durante todo este proceso de la instauración de las bibliotecas, la labor realizada por la bibliotecaria, filóloga y lexicógrafa María Juana Moliner Ruiz, quien estuvo al frente de la Delegación de las Misiones Pedagógicas de Valencia. Ella organizó una red bibliotecaria a partir de 115 bibliotecas ya establecidas en 1935, también, contribuyó en la creación de la Escuela Normal de Maestros, donde instauró que los graduados pudieran realizar prácticas para formarse en la labor bibliotecaria y ser capaces de trabajar en las bibliotecas de la red. Desde los hechos anteriores hasta la actualidad, las bibliotecas públicas han continuado su camino de inserción en la sociedad española contribuyendo a la construcción de espacios educativos y culturales para la ciudadanía.

Las anteriores se establecieron de acuerdo a un convenio firmado entre el Ayuntamiento de cada ciudad y la Consellería de Cultura del Gobierno de la Comunidad Valenciana. Será cada Ayuntamiento quien vele por la asignación del presupuesto para el financiamiento de dichas unidades de información geográficamente ubicadas dentro de la Casa de la Cultura de cada localidad.

En específico, respecto a la Biblioteca de Simat se establece oficialmente en 1983, cuando se firma con la Conselleria de Cultura un convenio de colaboración, donde se recibe un lote de libros, que se incorpora a otros existentes, y se estipula la contratación de una bibliotecaria, con un horario regular de apertura al público de 15 horas semanales distribuidas de lunes a viernes. Y a finales de 1988, se inaugura la actual Casa de la Cultura, y la biblioteca se sitúa en el primer piso del edificio.

En diciembre del 2002, la Biblioteca forma parte del Catálogo Colectivo de las Bibliotecas de la Xarxa de Lectura Pública Valenciana, y desde su creación en la década de 1980, su objetivo primordial ha sido proyectar a las bibliotecas como centros de información y de acercamiento a la cultura 
local. La Biblioteca de Simat se conoce como una Biblioteca Pública o "rural", término con el cual se denomina también a dichas bibliotecas, pertenece al Municipio de Simat de la Valldigna, situado en la Comarca de la Valldigna y atiende a una población de alrededor de 3.500 habitantes. Concurren a dicha Biblioteca desde bebés hasta adultos mayores, por lo tanto, se atiende a una población de usuarios muy diversa en horarios de 8:00 a.m. a 1:30 p.m. y de 4:00 p.m. a 8:00 p.m. de lunes a viernes y los sábados de 9:00 a.m. a 1:30 p.m. Actualmente, la colección de la biblioteca cuenta con cerca de 13.500 volúmenes.

El personal está conformado por una profesional en biblioteconomía y dos asistentes que trabajan en turnos alternos durante la semana. La biblioteca se encuentra ubicada en la primera planta de la Casa de la Cultura. En la entrada del edificio dispone de una sala de exposiciones y reuniones, en el primer piso está la sala general de lectura, la zona de préstamo y atención al público y un espacio destinado a la sección infantil. En el segundo piso está ubicada la sala que alberga la sección local y depósito de materiales, hay también un aula para uso de estudio individual y donde se realizan talleres, además de estar ubicada la sala de reuniones de la Asociación de Amas de Casa, y en el cuarto piso se encuentra un auditorio con capacidad para 150 personas, donde se realizan talleres de animación lectora, obras de teatro y otras actividades recreativas.

\section{LA PROYECCIÓN DE LA BIBLIOTECA PÚBLICA HACIA LA COMUNIDAD}

La finalidad de la Biblioteca Pública está enfocada en facilitar los recursos de información y servicios que puedan contribuir a mejorar la vida de las personas a nivel integral, brindando un espacio de apertura que fomente en el colectivo, progreso y desarrollo enfocado en la adquisición de nuevos conocimientos. De acuerdo a la IFLA, la Biblioteca Pública se define como una organización:

Establecida, respaldada y financiada por la comunidad, ya sea por conducto de una autoridad u órgano local, regional o nacional, o mediante cualquier otra forma de organización colectiva. Brinda acceso al conocimiento, la información y las obras de la imaginación gracias a toda una serie de recursos y servicios y está a disposición de todos los miembros de la comunidad por igual, sean cuales fueren su raza, nacionalidad, edad, sexo, religión, idioma, discapacidad, condición económica y laboral y nivel de instrucción. (IFLA, 2001, p.8)

En Simat de la Valldigna, la Biblioteca Pública constituye un espacio de servicio de mucha demanda por parte de los ciudadanos que cada vez más solicitan nuevos servicios y donde se hace necesario satisfacer sus requerimientos de información, por medio de propuestas accesibles e innovadoras. Asimismo, se da gran relevancia a la conservación del patrimonio cultural, como una parte fundamental de la preservación de la memoria y la identidad de las poblaciones que conforman dicha comunidad. La biblioteca como agente social se constituye en un espacio ciudadano que, adecuándose a los nuevos tiempos, busca propiciar el desarrollo social y cultural de las comunidades, dentro del marco de una sociedad democrática e inclusiva.

En tal caso, la Biblioteca Pública de Simat pretende, por medio de nuevos 
proyectos e iniciativas, potenciar su valor como agente en constante evolución, coexistiendo y proyectándose como un espacio dialéctico y abierto a la confluencia de distintos saberes que en su conjunto buscan promover nuevos conocimientos.

Dicha Biblioteca participa con frecuencia y de forma más activa en el fomento de la alfabetización, enfrentando constantes desafíos respecto a la colaboración y la formación de alianzas, como por ejemplo: con las asociaciones de vecinos que utilizan la Casa de la Cultura que forma parte de la Biblioteca para el planeamiento y ejecución de distintos talleres y actividades a nivel comunal; las subvenciones proporcionadas por las diferentes autoridades gubernamentales y la falta de más personal bibliotecario y técnicos culturales que apoyen su agenda cultural, lo que constituye para la bibliotecaria a cargo un reto constante de proyección a nivel laboral y social, en cuanto a la organización de escenarios de aprendizaje participativos que tengan permanencia e incidan de forma positiva en la vida cotidiana de los ciudadanos.

Así mismo, se caracteriza por ciertos elementos muy propios, debido al servicio público que presta jugando el Estado un papel preponderante en la facilitación de los medios para su instalación, mantenimiento y servicios que prestará a los ciudadanos miembros de la comunidad donde se encuentra inserta, esto conlleva a que un órgano de dirección y coordinación, sea el encargado de llevar un control de los servicios bibliotecarios y de la estructura organizativa de la Biblioteca por medio de una normativa que permita su adecuado funcionamiento, dentro de un marco común de directrices que, a la vez, faciliten la organización y la extensión los servicios de la Biblioteca en un ámbito geográfico determinado.

Actualmente, las Bibliotecas Públicas no pueden concebirse como un ente aislado y sin trascendencia en la comunidad, todo lo contrario, deben ser un nuevo modelo que tenga una especial importancia y repercusión en la vida de los ciudadanos. Se ha convertido en un lugar de acogida que desarrolle un papel activo y preponderante, donde existe colaboración con las distintas instituciones y fuerzas vivas de la comunidad. Debe ser, además, una institución que desarrolle tanto actividades de carácter cultural y formativas ya tradicionales en las Bibliotecas Públicas como también actividades de ocio y recreación que le permitan proyectarse de forma dinámica, para actuar como un centro de información donde sea un órgano de participación muy activo en la sociedad y junto a los ciudadanos.

Estas acciones de integración y diversificación requieren de un intercambio constante de ideas y de experiencias, de un trabajo en alianza tanto con las distintas instituciones estatales y privadas, como con los propios ciudadanos que al fin y al cabo son el universo, la comunidad de usuarios que da vida a la Biblioteca y que potencia su desarrollo social en constante cambio en la comunidad. Lo anterior tiene que ser un proceso continuo y constructivo que permita la obtención de logros que se proyecten a los lectores, como una fuerza viva e instrumento indispensable para fomentar en las personas espacios dinamizadores enmarcados en los campos del saber, el disfrute y la cultura.

\section{LA SECCIÓN DE TEMAS LOCALES}

Tanto las casas de la cultura, los archivos y los museos cumplen con una 
serie de necesidades específicas con distintas finalidades y objetivos bien definidos respecto a la difusión y salvaguarda de materiales, que buscan poder satisfacer las necesidades de información relacionadas con la cultura local de una comunidad en particular. Pero esencialmente por su dimensión y carácter cultural es la Biblioteca Pública la llamada a realizar esta labor; al respecto, la Federación Internacional de Asociaciones de Bibliotecarios y Bibliotecas (IFLA) hace referencia a la importancia de que sea esta institución quien tenga a su cargo la sección de temas locales. En su manifiesto se refiere a la cultura local de la siguiente manera:

La biblioteca pública debe ser la institución fundamental de la comunidad en la que se encuentra en lo que se refiere al acopio, la preservación y la promoción de la cultura local en todas sus modalidades. Puede hacerlo de diferentes maneras, por ejemplo, manteniendo los fondos relativos a la historia del lugar, organizando exposiciones y narraciones orales, editando publicaciones de interés local y creando programas interactivos sobre temas locales. (IFLA, 2001, p. 14)

Dentro de este rol de preservación y promoción de la cultura en sus distintas particularidades, juega un papel relevante la colección de temas locales en la Biblioteca Pública que cubre tópicos de interés para la comunidad, abarcando su pasado, su presente y su futuro, que Díaz Grau y García Gómez definen como:

El conjunto de documentos propios de una biblioteca que tratan temas de interés local de valor para el conocimiento del pasado, presente y perspectivas de futuro de la localidad. Esta colección forma la sección local y sus documentos, que pueden ser primarios, secundarios o terciarios, y/o que pueden estar en posesión de la biblioteca o estar prestados o estar cedidos a ella, son adquiridos, organizados, conservados comunicados y puestos a disposición de los usuarios, con la pretensión de facilitar su acceso, uso y conocimiento entre la comunidad. $(2005$, p. 29)

De esta forma, la sección de temas locales de la Biblioteca de Simat tiene como finalidad recoger, conservar y organizar materiales bibliográficos, hemerográficos, realias, materiales sonoros y audiovisuales que son de interés local para el colectivo, debido a que las colecciones existentes en esta sección poseen peculiares características de información contenida, lo que las hace poseedoras de un referente importante de aprendizaje local.

Los documentos de dicha sección están ordenados siguiendo en sistema de Clasificación Decimal Universal (CDU) y las Reglas de Catalogación del Ministerio de Cultura Español. La colección está dividida de la siguiente manera:

\subsection{Libros históricos}

Se encuentran aquí libros cuya característica común es poseer antiguos cuños que se usaban en las bibliotecas a partir de la Guerra Civil Española en 1936. Además, a principios del año 2017 se adquirió la colección de libros del historiador local Josep Toledo Girau, quien murió en 1976, en la cual se pueden encontrar libros recopilados por él y que datan de 1669. Resalta la 
importancia, ya que el Sr. Toledo fue un gran investigador de la población de Simat y la Comarca de la Valldigna. En sus escritos se encuentra información muy valiosa sobre diversos aspectos culturales de dicha región (ver Figura 1)².

\section{FIGURA 1}

Libro de la colección personal del historiador Josep Toledo Girau, 2017

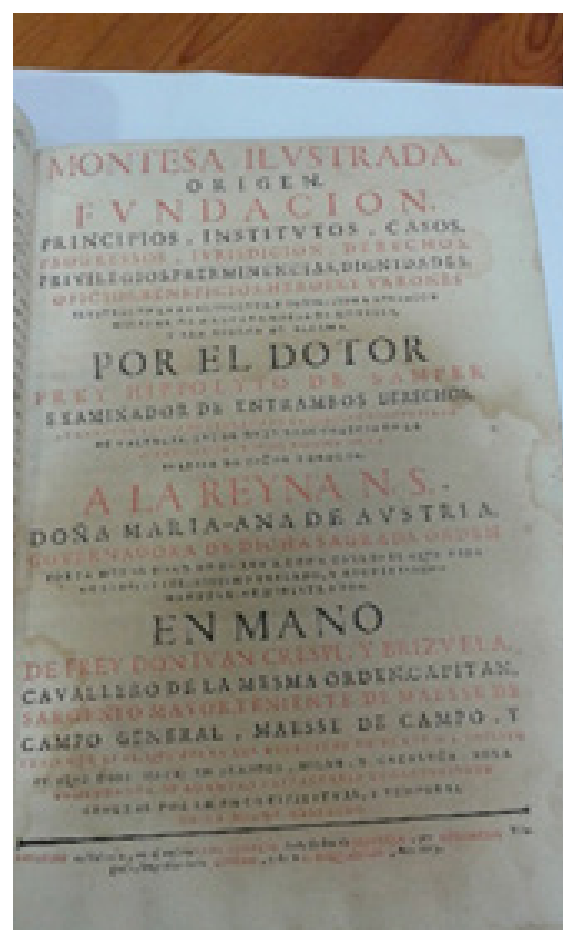

Fuente: Fotografía tomada por la autora de la Colección Local Biblioteca Pública de Simat.

\subsection{Monografías}

En esta sección se encuentran monografías sobre la historia de Simat escritas por cualquier autor, ya sea oriundo de la comunidad o trabajos de autores nacidos propiamente en la comunidad y que escriben sobre muy diversos temas.

\subsection{Publicaciones Seriadas}

Las que se encuentran en esta sección poseen la característica de incluir temáticas comunales, algunas de ellas fueron producidas por colectivos sociales, como por ejemplo, "Simat de Casa en Casa" elaborada por los vecinos de Simat. (ver Figura 2).

2 Todas las fotografías presentadas en este documento fueron realizadas con la autorización de las autoridades de la Biblioteca Pública de S. 
FIGURA 2

Publicación llamada "Simat de casa en casa", realizada por un grupo de vecinos de Simat, 2017.

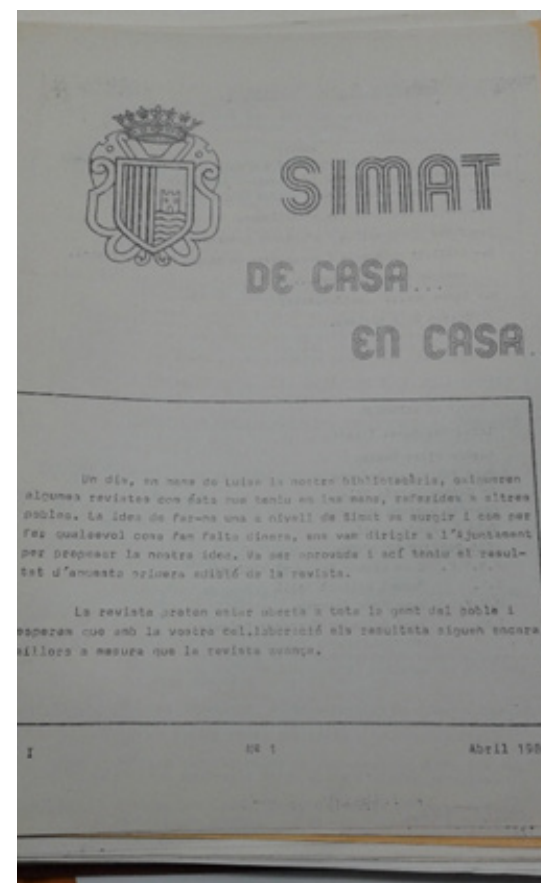

Fuente: Fotografía tomada por la autora de la Colección Local Biblioteca Pública de Simat.

Se ubican aquí los "libros de fiestas", que corresponden a una serie de folletos que seguían una secuencia en su edición y en los cuales se informaba a la población sobre las distintas celebraciones locales de índole religiosa celebradas en la localidad, como por ejemplo, la fiesta a San Antonio, Santa Ana y la Inmaculada Concepción. Se han incluido, a su vez, programas de fiestas pertenecientes a la pedanía (barrios que pertenecen a Simat pero geográficamente se encuentran ubicados en otra zona) como la población de Pla de Corrals.

\subsection{Materiales gráficos}

Desde hace varios años, la Biblioteca ha realizado una campaña para instar a los ciudadanos a que donen o presten las fotografías antiguas que posean, con este aporte ha cooperado también el Ayuntamiento, quien posee un fondo documental; estas fotografías reflejan una realidad observada, donde se retrata un momento muy particular, generando una imagen que es el recuerdo de ese momento irrepetible.

Las fotografías recopiladas se encuentran organizadas por:

- Oficios: se refieren a personas que trabajaban como músicos, agricultores, pintores, conserjes, panaderos, electricistas, obreros y cocineros, entre otros.

- Acontecimientos religiosos: como bautizos, comuniones, confirmaciones, matrimonios y procesiones religiosas.

- Actos festivos no religiosos: como bailes de disfraces, fiestas locales o patronales como cordà, correfocs (espectáculos de pólvora con cohetes), bous al carrer (corridas de toros), ferias, mercados. 
- Fotografías paisajistas: sobre diferentes calles, edificios como el Ayuntamiento, el mercado, el cine, la casa de la cultura, los lavaderos públicos, el cementerio y el calvario. Es posible ver distintos sitios como las fuentes (Font Gran, Font Menor, Font Tarda, Font del Cirer), El Raconet (un huerto), Pla de Corrals (pedanía) y Les Foies (barrio) (ver Figuras 3 y 4 )

FIGURA 3

Calle "Plaza de la Iglesia" en el centro de la ciudad, en 1952

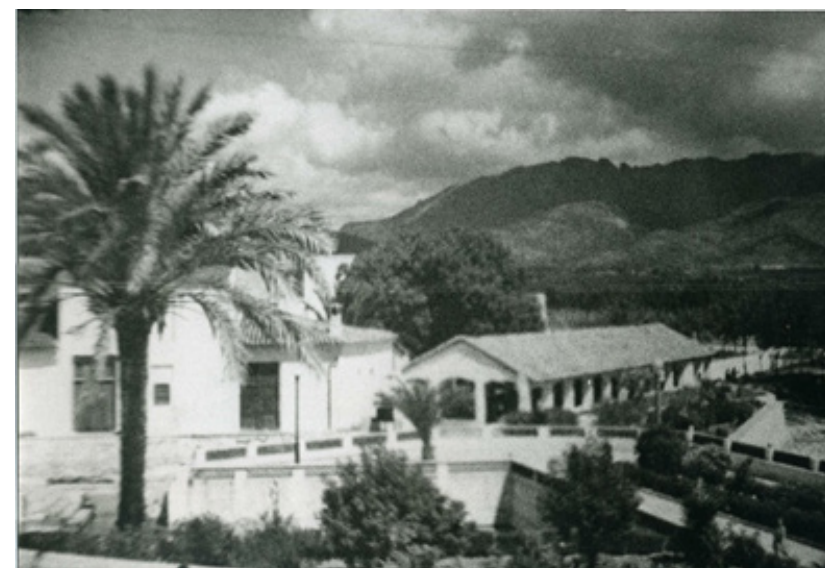

Fuente: Fotografía tomada por la autora de la Colección Local Biblioteca Pública de Simat.

FIGURA 4

Lavaderos de la ciudad de Simat en 1955

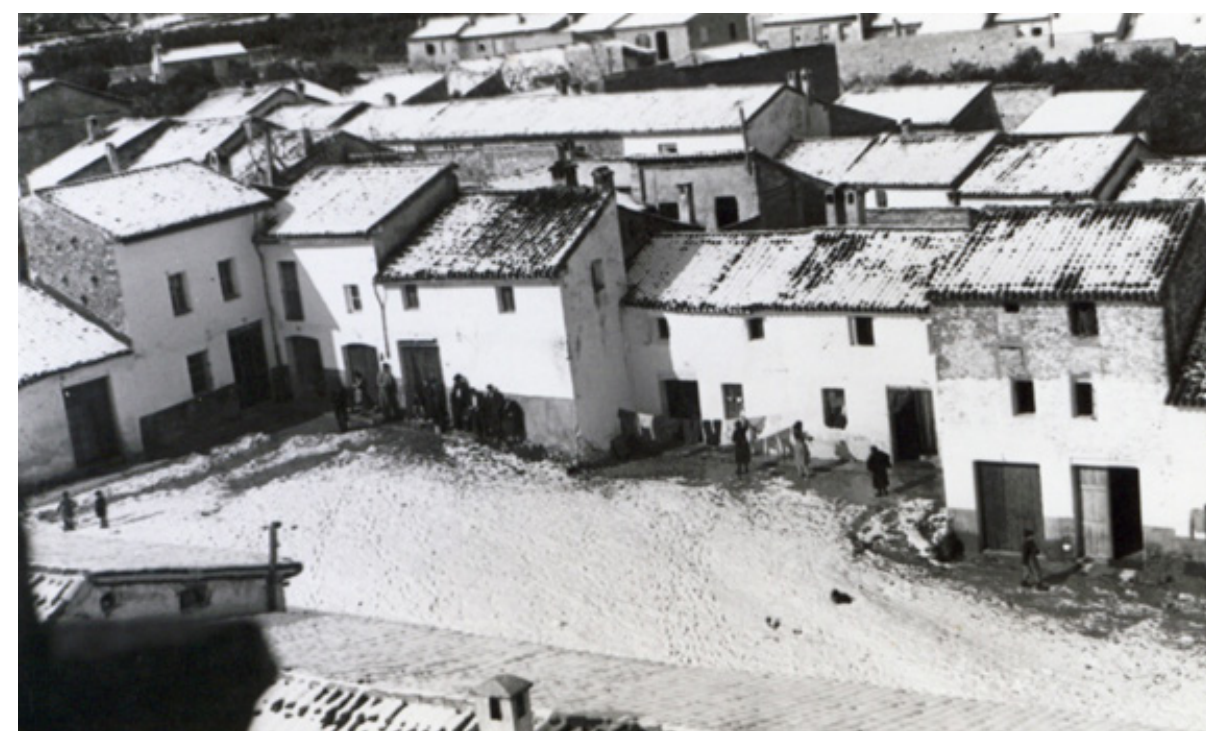

Fuente: Fotografía tomada por la autora de la Colección Local Biblioteca Pública de Simat. Nota: Se observa al fondo a la derecha uno de los antiguos lavaderos techados de la ciudad.

- Retratos de estudio: en este apartado las familias aportan retratos de niños, hombres, mujeres, parejas de novios y esposos (ver Figuras 5 y 6).

- Deportes: incluye diferentes deportes como "joc de la pilota" (juego de pelota), atletismo, caza, juegos de mesa, natación y motocros. 
FIGURA 5

Escena de una familia de la comunidad portando sus vestidos tradicionales en 1941

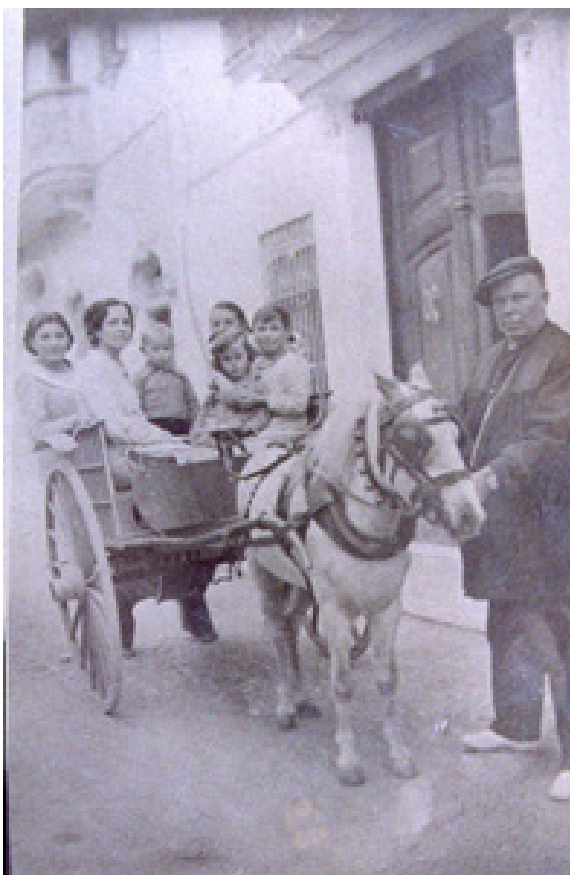

Fuente: Fotografía tomada por la autora de la Colección Local Biblioteca Pública de Simat

FIGURA 6

Foto de estudio de señoras residentes en Simat en 1892

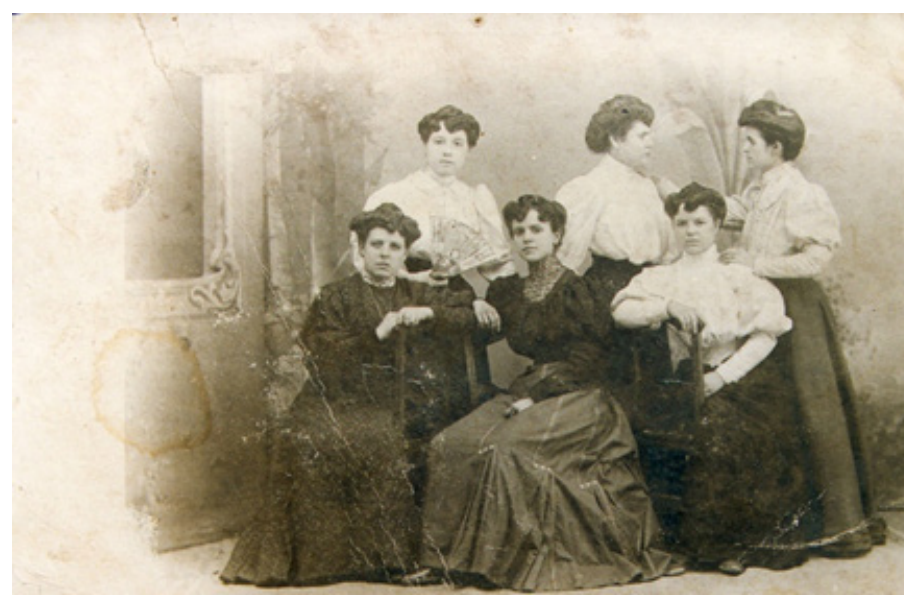

Fuente: Fotografía tomada por la autora de la Colección Local Biblioteca Pública de Simat.

- Grupos de escolares: fotos de los niños en la Escuela Pública de la localidad (ver Figura 7).

- Carteles de anuncios: corresponde a todo tipo de carteles relacionados con acontecimientos o actividades de la vida cotidiana de la población (ver Figura 8). 
FIGURA 7

Niños de la escuela pública de la comunidad en la Antigua estación de tren de la Valldigna, 1947

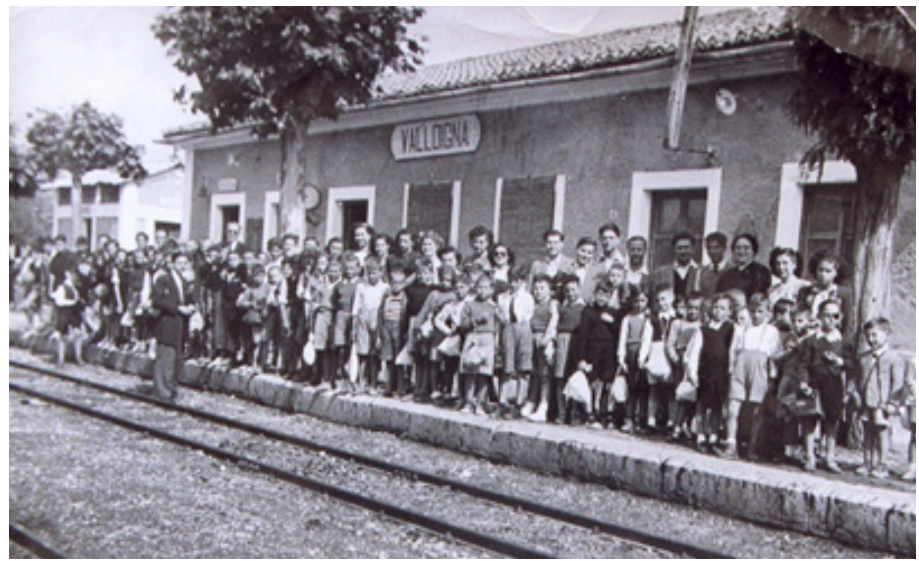

Fuente: Fotografía tomada por la autora de la Colección Local Biblioteca Pública de Simat.

FIGURA 8

Cartel con anuncios de actividades culturales de 1993

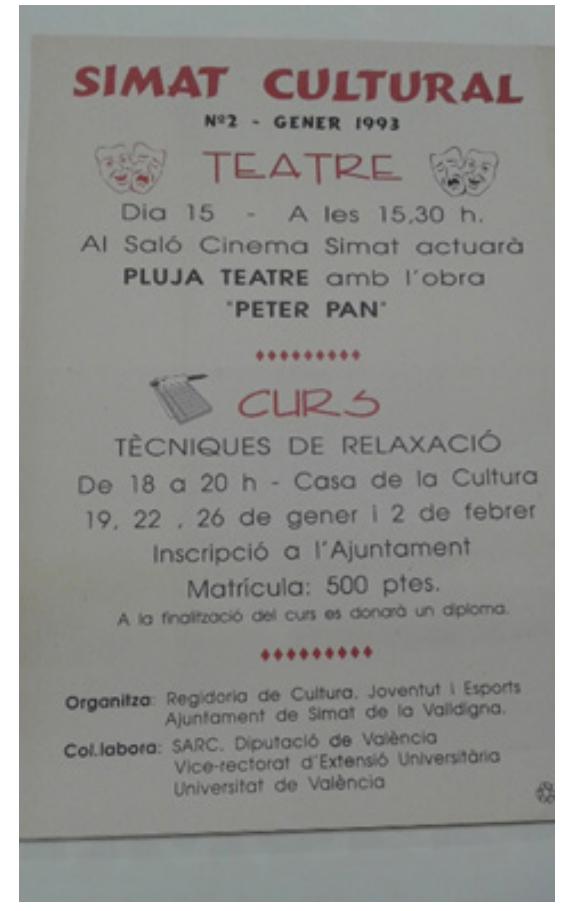

Fuente: Fotografía tomada por la autora de la Colección Local Biblioteca Pública de Simat.

- Calendarios: se recopilan los calendarios producidos por diferentes empresas, asociaciones y del Ayuntamiento, ya que son ilustrados, la mayoría de las veces, con fotografías representativas de la vida cultural de la comunidad, como deporte, música, y vistas de la naturaleza.

- Folletos: se contemplan los relacionados con ventas de apartamentos, ofertas de los comercios, anuncios de actividades culturales como exposiciones artísticas o fiestas de la Iglesia. 


\subsection{Realias}

En este apartado se conservan todos aquellos materiales promocionales que se hacen para actividades importantes en la localidad, no solo los generados a nivel del propio Ayuntamiento, sino también los producidos por las diferentes asociaciones comunales, como por ejemplo, bolígrafos, carteras, llaveros, camisetas, bolsas, tazas y gorras (ver Figura 9).

\section{FIGURA 9}

Materiales de promoción de la Biblioteca de Simat

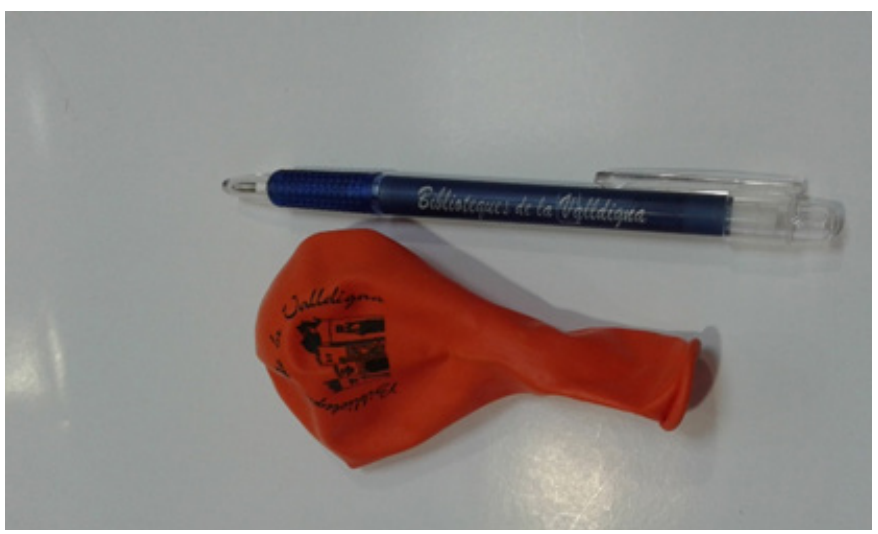

Fuente: Fotografía tomada por la autora de la Colección Local Biblioteca Pública de Simat.

\subsection{Juegos}

En este apartado se recolectan juegos de mesa, como el Juego de la Oca y El Trivall, estos juegos poseen la característica de estar hechos con temáticas ambientadas a la Comarca de la Valldigna (ver Figura 10).

FIGURA 10

Juego temático de la Oca sobre la población de Simat

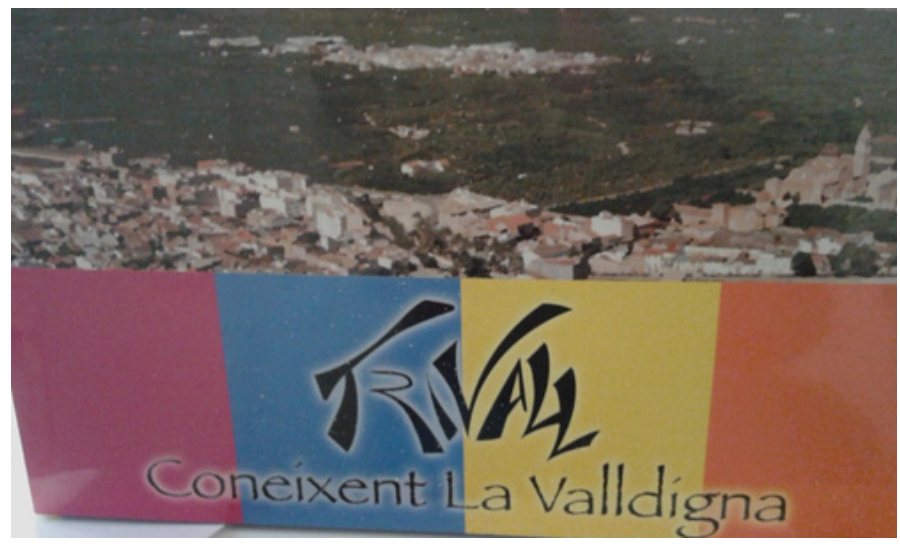

Fuente: Fotografía tomada por la autora de la Colección Local Biblioteca Pública de Simat Nota: En la fotografía se presenta la tapa del juego, donde se observa una foto panorámica de Simat. 


\subsection{Dibujos}

Se recopilaron dibujos en una actividad conjunta que se realizó una sola vez con personas de la localidad catalana de Les Valls d'Aneu; también se localizan aquí carpetas con dibujos de escolares y de jóvenes colegiales que recogen temas comunales diversos, y los realizados por niños de la Escuela local, luego de asistir a algunos de los talleres de animación lectora que se hacen en la Biblioteca.

\subsection{Materiales sonoros}

Se encuentran entre ellos:

- Distintas Cintas de VHS, como por ejemplo:"700 anys d'història. Santa Maria de Valldigna", un relato histórico de la comunidad y"Fem Fredat, Colla de dimonis de Simat de la Valldigna" (Grupo de demonios que dan miedo), este corto relata el trabajo que hace el grupo Fem Fredat de espectáculos artísticos con pólvora.

- Un DVD de la revista "La Safor en Xifres" (La Safor en cifras), programa referente a dicha revista.

- Dos cintas de casete del programa radiofónico que se realizaba en Simat llamado "Tramuntana", que se emitía hace unos 25 años.

- Un CD de música popular de la cantante de Simat Maria del Carmen Girau. Nacida en 1940 y quien en los años 60 dejó su carrera artística para continuar con su antigua profesión de farmacéutica.

- 30 casetes con entrevistas a personas nacidas entre los años 1920 y 1930, los cuales reconstruyen por medio del registro y recopilación de testimonios orales de los pobladores, distintos aspectos de la vida cotidiana en la localidad de Simat.

\subsection{Materiales especiales}

Esta sección tiene la peculiaridad de recuperar y conservar objetos representativos utilizados para distintas actividades tanto de la vida diaria de la comunidad, como de actividades conmemorativas, y una trompeta para anunciar eventos locales (ver Figura 11).

FIGURA 11

Trompeta para llamar a los ciudadanos y anunciar noticias locales

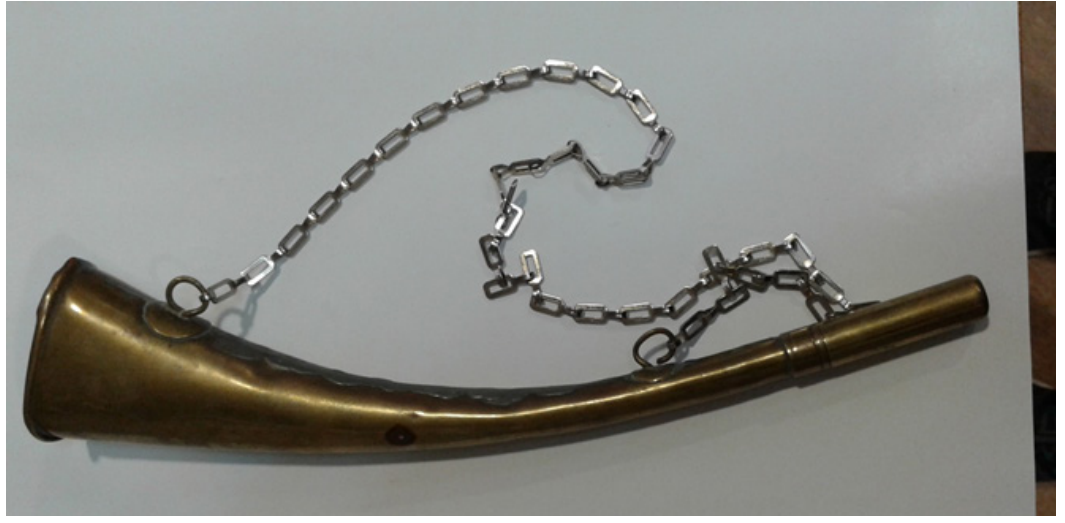

Fuente: Fotografía tomada por la autora de la Colección Local Biblioteca Pública de Simat. 
Entre otros materiales, se presentan documentos de identidad y otros similares de ciudadanos de la localidad, tales como:

- Libreta de identidad de la ciudadana Carmen Mogort. Expedida en 1938. Documento oficial de identidad otorgado por el gobierno español de la época.

- Un certificado de buena conducta de Joaquín Llacer Codina vecino de la comunidad, el cual era necesario para conseguir un pasaporte en 1956.

- Una notificación para Joaquín Llacer de 1936, en la cual se le solicita su incorporación para enlistarse en el ejército.

- Una subscripción a la "Revista Social del Sagrado Corazón" por un valor de 50 pesetas, a nombre de la señora Adelina Valls Gómez.

- Un boleto del 1960 del autobús la "Simatense" por un valor de 4 pesetas. Este transporte realizaba la ruta de Simat a Tavernes de la Valldigna.

- Un contrato de 1955-1956, para una persona que fue empleada en una de las compañías productoras y recolectoras de naranjas, uno de los cultivos más importantes de la zona.

- Una muestra de una esquela funeraria. (ver Figura 12)

FIGURA 12

Esquela del cura párroco de la comunidad don Vicente Llacer Solares

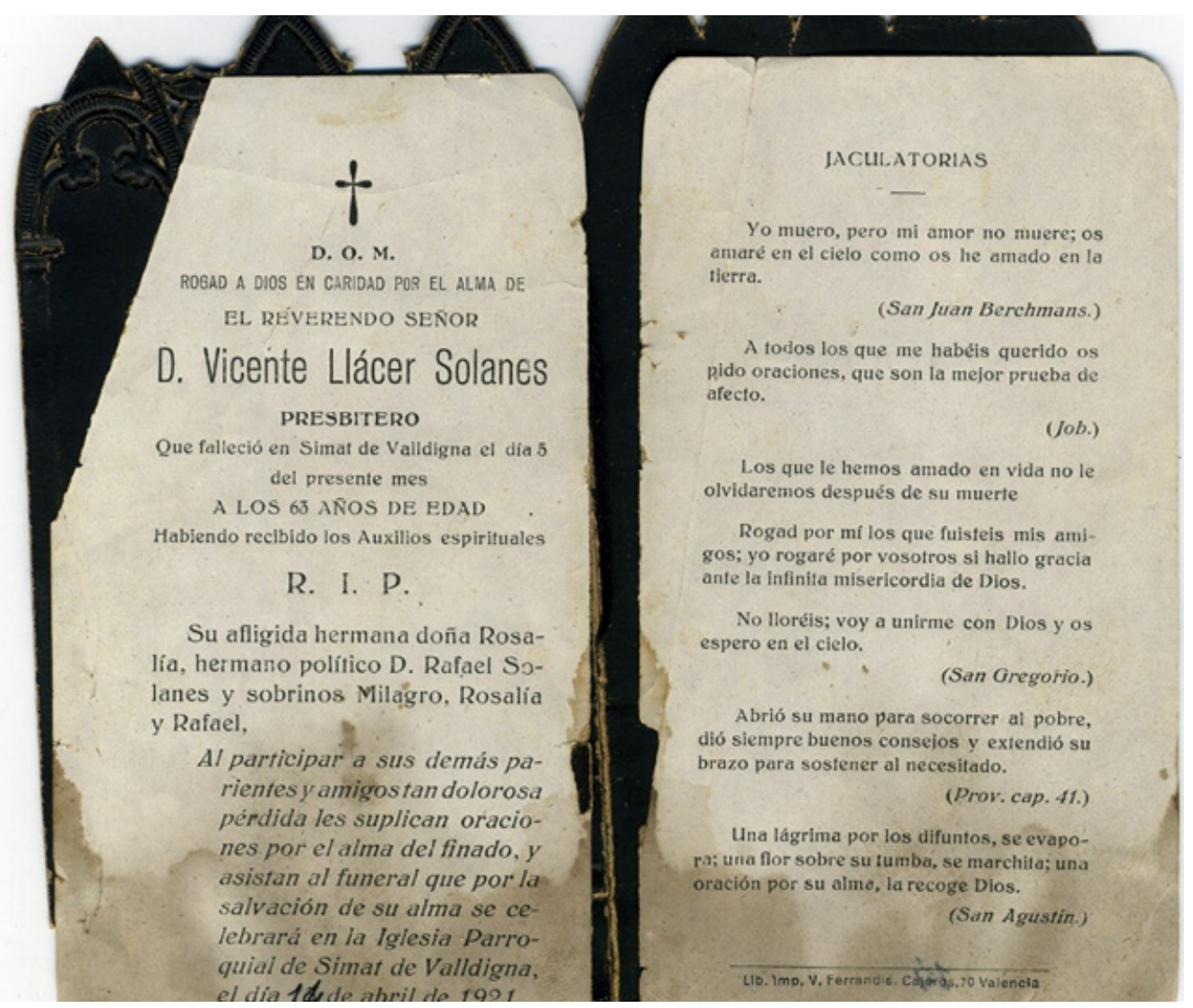

Fuente: Fotografía tomada por la autora de la Colección Local Biblioteca Pública de Simat. 


\section{CONCLUSIÓN}

La sección de temas locales de la Biblioteca Pública aporta a la comunidad de Simat de la Valldigna un patrimonio de información imprescindible, para la reconstrucción y preservación de la identidad histórico cultural de dicha población y su aporte está enfocado en brindar al usuario fuentes de información diversas sobre distintos aspectos de la vida en la zona, lo cual contribuye a preservar la memoria del pasado por medio del acopio, la conservación y el acceso a materiales relacionados con todas aquellas manifestaciones culturales sobre diversas temáticas referentes a la comunidad.

El interés por la colección de temas locales adquiere una dimensión especial cuando se dinamiza y se logra proyectar al máximo dicha colección, mediante actividades donde participe la Biblioteca de forma activa dando a conocer su potencial de recursos de información.

Respondiendo en función de las demandas de la población la Biblioteca de Simat como centro cultural de la comunidad debe considerar como primordial objetivo la responsabilidad y el papel que tiene que cumplir el fondo local frente a las necesidades de los distintos grupos sociales que harán uso de sus servicios, con el afán de aprender más acerca de su propia colectividad, lo cual implica el manejo y puesta en práctica de un adecuado uso de los recursos concernientes a financiación, tecnología, espacio físico, acervo de la colección y personal disponible para su adecuado funcionamiento.

De esta manera, será posible asegurar una gestión adecuada con continuidad de los servicios que respondan a prácticas y procedimientos para satisfacer las necesidades de los usuarios, de lo contrario, la sección local no podrá diseminar el conocimiento tan valioso acumulado en su fondo local y se convertirá en un simple depósito de materiales. La proyección y la utilidad práctica de la sección de temas locales de la biblioteca pública significa dar valor a las raíces que representan la memoria de los antepasados, que con sus testimonios han construido un amplio abanico de historias dando vida y perpetuidad a un colectivo en particular. 


\section{REFERENCIAS}

Canet, X. (1999). A la meua terra. (17 poemes i un relat). Valencia, España: Imprenta Reyes.

Díaz Grau, A. y García Gómez, F. J. (2005). La colección local en la biblioteca pública (I) concepto, delimitación y justificación. Boletín de la asociación Andaluza de Bibliotecarios, 20(78), 19-42. Recuperado de https://dialnet.unirioja.es/servlet/articulo?codigo $=1356543$

Federación Internacional de Asociaciones de Bibliotecarios y Biblioteca, y Organización de las Naciones Unidas para la Educación, la Ciencia y la Cultura. (abril, 2001). Directrices IFLA/UNESCO para el desarrollo o servicio de Bibliotecas públicas. Federación Internacional de Asociaciones de Bibliotecarios y Bibliotecas. Recuperado de https://www.ifla.org/files/ assets/hq/publications/archive/the-public-library-service/pg01-s.pdf

Ley Orgánica 5/1982, de 1 de julio, de Estatuto de Autonomía de la Comunidad Valenciana. (10 de julio de 1982). Recuperado de https:// www.boe.es/buscar/act.php?id=BOE-A-1982-17235

Ripoll, L. (2015). Simat de Valldigna 1915 [Edición de Elia Saneleuterio]. Valencia: Ajuntament de Simat de la Valldigna. 


\section{e $\begin{aligned} & \text { e-Ciencias de la } \\ & \text { Información }\end{aligned}$}
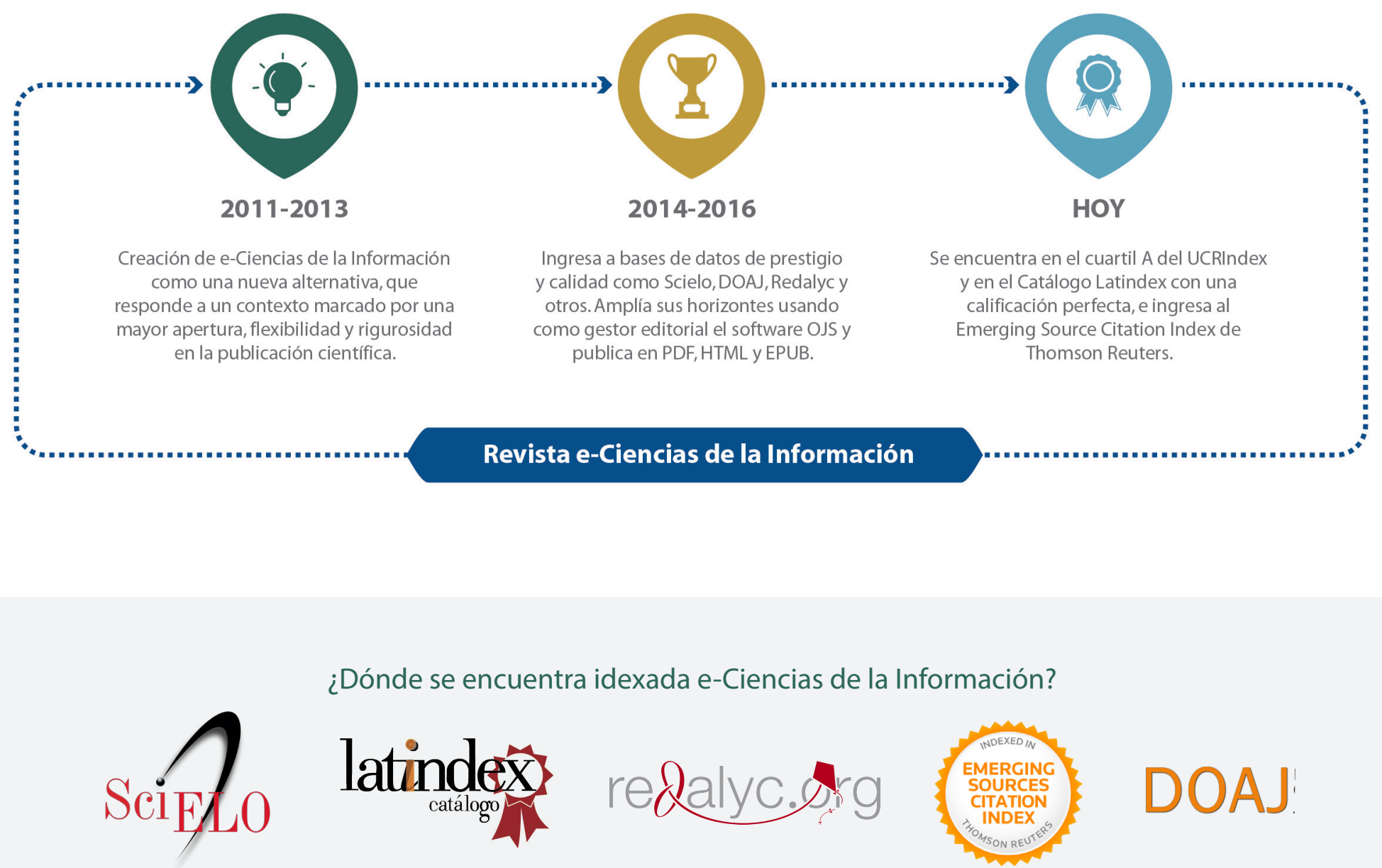

¿Dónde se encuentra idexada e-Ciencias de la Información?
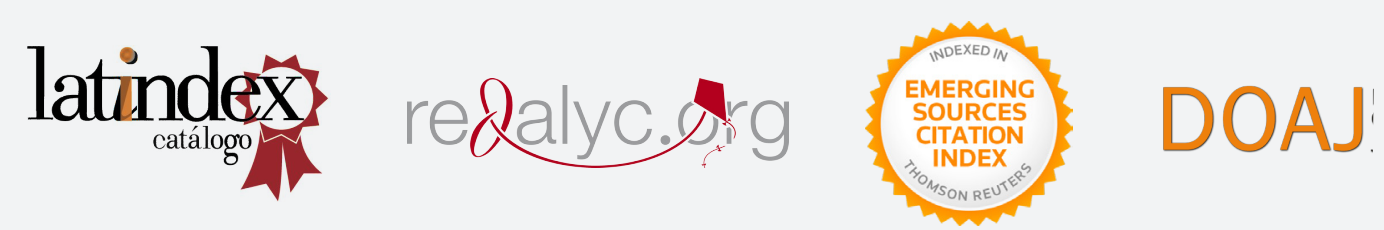

Para más información ingrese a nuestra lista completa de indexadores

¿Desea publicar su trabajo?

Ingrese aquí

O escríbanos a la siguiente dirección

revista.ebci@ucr.ac.cr 